\title{
Study of the relation between biliary cirrhosis and hepatopulmonary syndrome and its reversibility among young rats submitted to common bile duct ligation
}

\author{
Leonardo Ervolino Corbi ${ }^{1}$, Maria Julia de Aro Braz ${ }^{1}$, Ana Cristina Aoun Tannuri², \\ Maria Cecília de Mendonça Coelho ${ }^{3}$, Suellen Serafini ${ }^{3}$, \\ Josiane de Oliveira Gonçalves ${ }^{3}$, Uenis Tannuri²
}

$\mathrm{T}$ he study entitled "Study of the relation between biliary cirrhosis and hepatopulmonary syndrome and its reversibility among young rats submitted to common bile duct ligation" aimed to deepen pathophysiological knowledge about one unpredictable complication of cirrhosis: the hepatopulmonary syndrome (HPS). Biliary atresia (BA) is an important cause of biliary cirrhosis in childhood, accounting for the majority of liver transplants and deaths due to liver failure ${ }^{1}$. Untreated, children with BA do not live beyond the first two years of life due to portal hypertension and hepatic failure, complications of the disease - even submitted to surgical intervention, many patients evolve to liver fibrosis and eventually cirrhosis. Liver transplant is frequently necessary: BA accounts for $60 \%$ of pediatric transplants ${ }^{2}$. One complication of cirrhosis is HPS, which is defined by the presence of liver disease or portal hypertension and pulmonary intravascular dilation causing abnormal alveolar-arterial oxygen gradient ${ }^{3}-$ it is present in 5 to $32 \%$ of cirrhotic patients ${ }^{4}$ and in up to $20 \%$ of children with $\mathrm{BA}^{5}$. The onset of HPS indicates poor prognosis and higher mortality in these groups of cirrhotic patients.
The current knowledge on the pathophysiology of the HPS is based mainly on experimental models of common bile duct ligation in rats and is still shallow. This model showed that HPS is due to increase in endothelial nitric oxide synthase (eNOS) expression, with larger production of nitric oxide (NO) and consequent vasodilation. The increased eNOS expression is secondary to the production of endothelin-1 (ET-1) by cholangiocytes of the cirrhotic liver and activation of ETb receptors in the pulmonary vascular endothelium. The natural history of HPS has not yet been fully described either, although its negative impact on the prognosis of the cirrhotic patient is already clear. Most patients may be asymptomatic or present dyspnea - classically, platypnea and orthodeoxia - with insidious onset. There may be vascular spiders, digital clubbing and cyanosis. None of these manifestations, however, is specific for the diagnosis of HPS $^{4,6}$. There is no clearly effective treatment for hepatopulmonary syndrome other than liver transplant, which is followed by remission of the disease in more than $80 \%$ of cases, especially in children ${ }^{3}$.

The objectives of this study were to determine how

\footnotetext{
$1^{\circ}$ Award Oswaldo Cruz, Surgical COMU 2017 - XXXVI Congresso Médico Universitário da Faculdade de Medicina da Universidade de São Paulo, Oct. 6-8, 2017.

1.Faculdade de Medicina, Universidade de Sao Paulo, Sao Paulo, SP, BR.Email: mjuliaarobraz@gmail.com, leonardo.corbi@hotmail.com. 2.Faculdade de Medicina, Universidade de Sao Paulo, Sao Paulo, SP, BR. Instituto da Criança, Hospital das Clínicas da FMUSP. Laboratório de Investigação em Cirurgia Pediátrica (LIM 30) - FMUSP. Email de contato: cristannuri@hotmail.com.

3. Laboratório de Investigação em Cirurgia Pediátrica (LIM 30) - FMUSP.

Responsável pela correspondência: Leonardo E. Corbi. Faculdade de Medicina da Universidade de São Paulo. Av. Doutor Arnaldo, 455, $4^{\circ}$ Andar, Sala 4106. Cerqueira César, São Paulo, SP, BR. Email de contato: leonardo.corbi@hotmail.com.
} 
long after common bile duct ligation HPS starts, correlating it with the degree of liver cirrhosis; to verify whether HPS is reversible after reconstruction of bile flow and for how long after bile duct ligation this procedure is effective; and to study further lung histological modifications in cirrhotic animals. 21-day-old Wistar rats were submitted to common bile duct ligation and allocated into groups A1-A5 and B1-B5. Animals in group A were sacrificed $2,3,4,5$ or 6 weeks after biliary obstruction, while those in group B were submitted to biliodigestive anastomosis $2,3,4,5$, or 6 weeks after the first procedure and sacrificed 3 weeks later. At the time of sacrifice, arterial blood was collected for analyses and samples from liver and lungs for histological and molecular analyzes. Liver and lung tissue were stained with hematoxylin-eosin. Gasometric parameters and expression in lung tissue of eNOS, NOS and endothelin (ET-1) were studied.

The results showed increased duct proliferation in liver tissue, as the time from biliary obstruction advanced. Also, from the total of 42 blood samples, 15 showed hypoxia $(\mathrm{pO} 2<85 \mathrm{mmHg}$ ) and 17 showed increased oxygen gradient ( $\mathrm{p}(\mathrm{A}-\mathrm{a}) \mathrm{O} 2>18 \mathrm{mmHg}$ ), although we found no clear correlation with time of obstruction or any of the molecular mediators analyzed. On the other hand, we observed several changes in lung tissue, including inflammation, thickening and vascular engorgement of alveolar septa, with apparent progression according to the time of biliary obstruction and hepatic injury in group A. The alveolar area appears to diminish in groups who underwent biliary obstruction for a longer time. Besides, we observed an increase in $\mathrm{p}(\mathrm{A}-\mathrm{a}) \mathrm{O} 2$ as time passes after obstruction in group A. In group $\mathrm{B}$, however, alterations in pulmonary parenchyma and arterial-alveolar oxygen gradient showed no impact of reestablishing biliary flow on the progression of lung damage.

Endothelin expression in lung tissue, interestingly, decreased as time of obstruction passed, both in group $\mathrm{A}$ and $\mathrm{B}$. Other studies have shown increased plasmatic endothelin in the common bile duct ligation model, shortly after ligation and not correlating with the time of obstruction. So, in the young rats we used, it is possible that the decrease in ET-1 lung expression is due to negative feedback of elevated plasmatic endothelin, secondary to the initial cholestatic stimulus. This stimulus may have been the most important factor in these alterations, since evolution of ET-1 expression is similar in groups A and B.

In conclusion, the model of common bile duct ligation is effective for causing liver disease and was able to create lung disease, as we found hypoxia, abnormal gas exchange and histologic alterations in a significant portion of our individuals. Nevertheless, the current study found no clear relation between alterations (molecular, histologic, and gasometrical) and period of biliary obstruction - with the exception of alveolar area in lung tissue - and could not find impacts of biliodigestive anastomosis on the onset or progression of lung disease. Further studies are necessary to understand the underlying mechanisms of HPS.

\section{REFERENCES}

1. Goldman M, Pranikoff T. Biliary disease in children. Cur Gastroenterol Reps. 2011;13(2):193-201. doi: 10.1007/s11894010-0169-1.

2. Gibelli N, Tannuri U, de Mello E, Rodrigues C. Bile duct ligation in neonatal rats: Is it a valid experimental model for biliary atresia studies? Pediatr Transplant. 2009;13(1):81-7. doi: 10.1111/j.1399-3046.2008.00947.x.

3. Zhang J, Fallon M. Hepatopulmonary syndrome: update on pathogenesis and clinical features. Nat Rev Gastroenterol Hepatol. 2012;9(9):539-49. doi: 10.1038/nrgastro.2012.123.

4. Barbé T, Losay J, Grimon G, Devictor D, Sardet A, Gauthier F, Houssin D, Bernard O. Pulmonary arteriovenous shunting in children with liver disease. J Pediatr. 1995;126(4):571-9. https://doi.org/10.1016/S0022-3476(95)70351-9

5. Rodriguez-Roisin R, Krowka MJ, Hervé P, Fallon MB; ERS Task Force Pulmonary-Hepatic Vascular Disorders (PHD) Scientific Committee. Pulmonary-hepatic vascular disorders (PHD). Eur Respir J. 2004;24(5):861-80. doi: 10.1183/09031936.04.00010904.

6. Gómez F, Martínez-Pallí G, Barberà J, Roca J, Navasa M, Rodríguez-Roisin R. Gas exchange mechanism of orthodeoxia in hepatopulmonary syndrome. Hepatology. 2004;40(3):660-6. doi: 10.1002/hep.20358.

Submitted for publication: Feb 22, 2018

Accepted in: Feb 26, 2018 